\title{
Current and upcoming pharmacotherapy for non-alcoholic fatty liver disease
}

\author{
Yaron Rotman, ${ }^{1}$ Arun J Sanyal ${ }^{2}$
}

${ }^{1}$ Liver and Energy Metabolism Unit, Liver Diseases Branch, National Institute of Diabetes and Digestive and Kidney Diseases, National Institute of Health, Bethesda, Maryland, USA

${ }^{2}$ Division of Gastroenterology, Hepatology and Nutrition, Department of Internal Medicine, Virginia Commonwealth University School of Medicine, Richmond, Virginia, USA

\section{Correspondence to}

Dr Yaron Rotman, Liver and Energy Metabolism Unit, Liver Diseases Branch, NIDDK, NIH, 10 Center Drive, Building 10, Room 9C434, MSC 1800, Bethesda, MD 20892-1800, USA; rotmany@niddk.nih.gov

Received 13 June 2016 Revised 29 August 2016 Accepted 30 August 2016 Published Online First 19 September 2016

\section{ABSTRACT}

Given the high prevalence and rising incidence of nonalcoholic fatty liver disease (NAFLD), the absence of approved therapies is striking. Although the mainstay of treatment of NAFLD is weight loss, it is hard to maintain, prompting the need for pharmacotherapy as well. A greater understanding of disease pathogenesis in recent years was followed by development of new classes of medications, as well as potential repurposing of currently available agents. NAFLD therapies target four main pathways. The dominant approach is targeting hepatic fat accumulation and the resultant metabolic stress. Medications in this group include peroxisome proliferator-activator receptor agonists (eg, pioglitazone, elafibranor, saroglitazar), medications targeting the bile acid-farnesoid $X$ receptor axis (obeticholic acid), inhibitors of de novo lipogenesis (aramchol, NDI010976), incretins (liraglutide) and fibroblast growth factor (FGF)-21 or FGF-19 analogues. A second approach is targeting the oxidative stress, inflammation and injury that follow the metabolic stress. Medications from this group include antioxidants (vitamin E), medications with a target in the tumour necrosis factor $\alpha$ pathway (emricasan, pentoxifylline) and immune modulators (amlexanox, cenicriviroc). A third group has a target in the gut, including antiobesity agents such as orlistat or gut microbiome modulators (IMM-124e, faecal microbial transplant, solithromycin). Finally, as the ongoing injury leads to fibrosis, the harbinger of liverrelated morbidity and mortality, antifibrotics (simtuzumab and GR-MD-02) will be an important element of therapy. It is very likely that in the next few years several medications will be available to clinicians treating patients with NAFLD across the entire spectrum of disease.

\section{INTRODUCTION}

Non-alcoholic fatty liver disease (NAFLD), defined as excess accumulation of fat in the liver, has become the most common cause for chronic liver disease in the Western world and is estimated to impact at least $30 \%$ of Americans ${ }^{1}{ }^{2}$ or Chinese $^{3}$ with the prevalence appearing to rise in recent years. ${ }^{45}$ Non-alcoholic steatohepatitis (NASH) is a subset of NAFLD, estimated to affect $2-5 \%$ of Americans, in which increased liver fat is accompanied by cellular injury, inflammatory infiltrate and, subsequently, liver fibrosis, which can progress to cirrhosis with its associated complications. ${ }^{6}$ Although fatty liver by itself is associated with other features of the metabolic syndrome such as obesity, diabetes mellitus type 2, hypertension and dyslipidemia, increased liver-related mortality is essentially limited to patients with NASH. ${ }^{7}$
Increased triglyceride deposition in the liver reflects an input/output imbalance of hepatic free fatty acid (FFA) metabolism. In obesity-associated NAFLD, there is an increase of FFA delivery to the liver, especially during the fed state, due to adipose tissue insulin resistance. ${ }^{8} 9$ In addition, de novo lipogenesis is increased, ${ }^{10}$ driven by the hyperinsulinemia as well as excess availability of carbohydrates. Compensatory increase in very low density lipoprotein (VLDL) secretion is not sufficient to overcome the excess formation of triglycerides ${ }^{11}$ while it is unclear whether $\beta$-oxidation is increased or decreased in these subjects. ${ }^{12}$

The accumulated triglycerides in steatosis appear to be relatively inert with benign outcome; hepatocellular injury is driven by lipotoxicity from FFAs and their derivatives, ${ }^{13}$ as well as overloading of mitochondrial capacity. This initial metabolic stress activates multiple cell stress pathways, including generation of reactive oxygen species, endoplasmic reticulum stress and apoptosis. Injury signals from stressed or dying hepatocytes, lipids and chemokines activate an immune response, including recruitment and activation of variety of immune cells, further increasing cellular injury. Key mediators are the Kupffer cells and macrophages, which are further activated by bacterial products from the gut microbiome.

Hepatocellular injury and immune cell activity converge to activate hepatic stellate cells, causing a change in their phenotype and deposition of collagen, resulting in increased fibrosis and hepatic architectural distortion.

Although the injury patterns are common and conserved, there is variability between patients with NAFLD in the degree of activation of each individual pathway, likely accounting for the heterogeneity of clinical phenotypes and severity. This may be secondary to different external stimuli (ie, dietary composition), genetic components and modulation by the gut microbiome, among other factors.

The remarkable progress that has been made in previous years in understanding disease pathogenesis has led to an explosion of medical therapies targeting various aspects of the fat accumulation and injury pathways. These can be grouped into four general classes (figure 1), according to their intended targets: (1) Medications with a primary metabolic target, geared to reduce hepatic fat accumulation and metabolic stress. (2) Medications addressing oxidative stress or the inflammation and injury components of NASH. (3) Medications with a primary gut target, modulating the interaction between the gut and the liver in NAFLD. (4) Antifibrotics, aiming to decrease the progressive fibrosis and resultant complications.
To cite: Rotman Y,
Sanyal AJ. Gut

2017;66:180-190. 


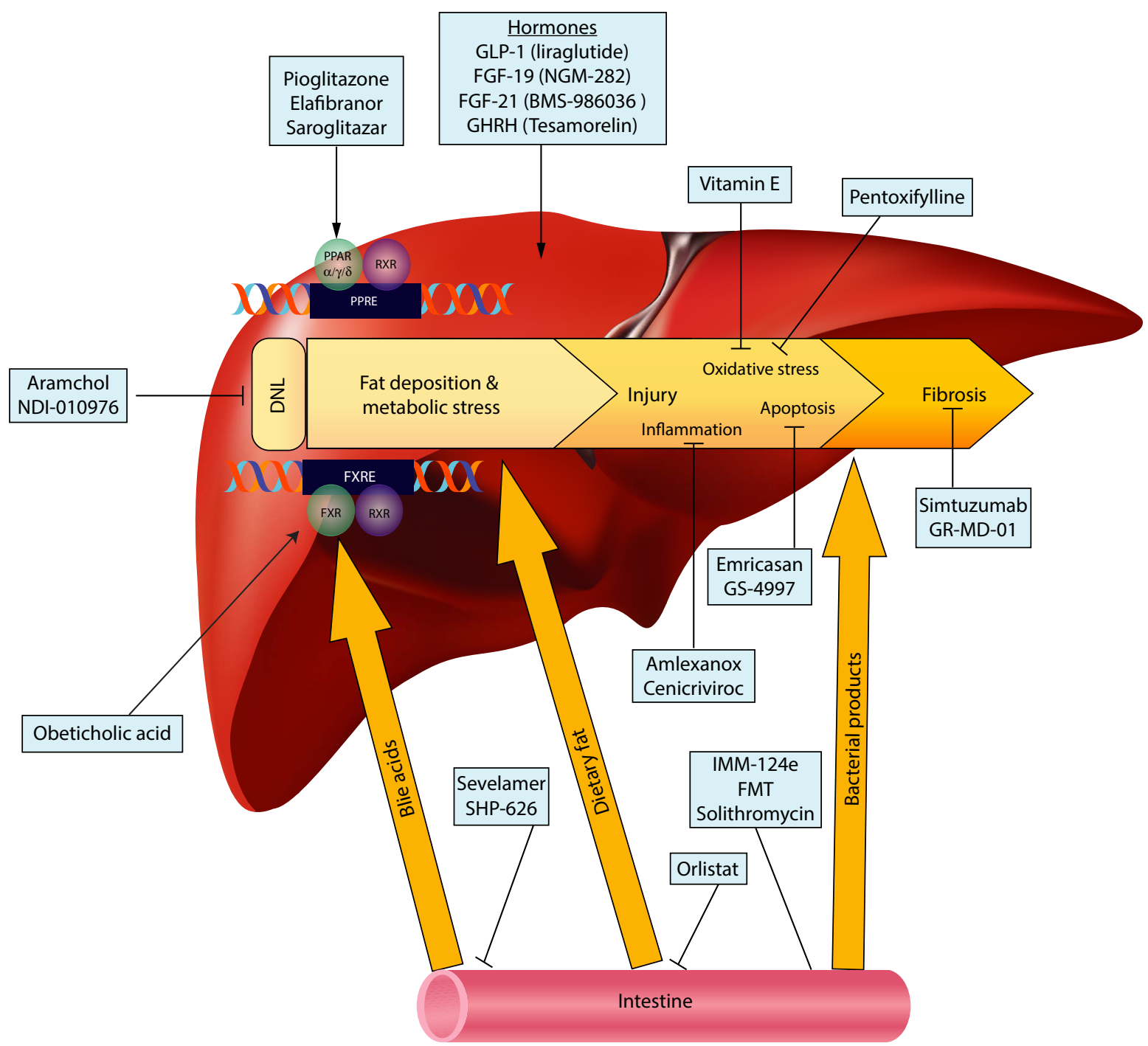

Figure 1 Targets of upcoming therapies for non-alcoholic fatty liver disease (NAFLD). DNL, de novo lipogenesis; FGF, fibroblast growth factor; FMT, faecal microbial transplant; FXR, farnesoid X receptor; FXRE, FXR response element; GHRH, growth hormone-releasing hormone; GLP-1, glucagon-like peptide-1; PPAR, peroxisome proliferator-activated receptor; PPRE, PPAR response element; RXR, retinoid X receptor.

\section{MEDICATIONS WITH A PRIMARY METABOLIC TARGET PPAR agonists}

The peroxisome proliferator-activator receptors (PPARs) are a family of nuclear receptors that bind a wide range of fatty acids and fatty acid derivatives and transcriptionally regulate a wide variety of metabolic processes (table 1 ). There are three PPARs $-\alpha, \beta / \delta$ and $\gamma$-that share the same target DNA sequence but differ in ligand selectivity and tissue distribution. ${ }^{14}$

PPAR $\alpha$ is expressed extensively in the liver, adipose tissue, heart, skeletal muscle and kidney; ${ }^{15}$ its activity increases in the fasting state and transcriptionally drives the expression of a large number of genes, including those regulating gluconeogenesis, $\beta$-oxidation, lipid transport and the hormone fibroblast growth factor (FGF)-21. In various animal models, PPAR $\alpha$ deletion, either at germline level ${ }^{16}$ or in hepatocytes only, ${ }^{17}$ is associated with worsening of hepatic steatosis. Fibrates, which are synthetic agonists of PPAR $\alpha$, are used extensively for clinical treatment of hypertriglyceridemia, but have not shown a consistent beneficial effect for the treatment of NAFLD, ${ }^{18}$ possibly related to their effect on $\operatorname{PPAR} \alpha$ outside the liver. PPAR $\delta$, another member of the PPAR family, has a wider expression distribution pattern, and beyond hepatocytes is also expressed in high levels in skeletal muscle and macrophages ${ }^{19}$ and its activation improves insulin sensitivity, decreases hepatic glucose production, increases fatty acid oxidation and decreases macrophage and Kupffer cell activation. PPAR $\delta$ activation has also been shown to decrease atherosclerotic disease in animal models. ${ }^{20}$ Treatment with a PPAR $\delta$ agonist in a pilot study decreased hepatic fat content, likely through an increase in fatty acid oxidation, ${ }^{21}$ but development has been halted due to safety concerns. Elafibranor (GFT-505) is a dual $\operatorname{PPAR} \alpha / \delta$ agonist, aiming to combine the beneficial effects of activating the two receptors. Animal data demonstrate that a beneficial effect of elafibranor on serum triglycerides, cholesterol and high density lipoprotein (HDL), and a reduction in hepatic fat that is mediated, at least in part, by non-PPAR $\alpha$-dependent mechanism. $^{22}{ }^{23}$ Post hoc analysis of short-term (4-12 weeks) phase II clinical trials using elafibranor for the treatment of metabolic syndrome demonstrated a significant reduction in ALT in subjects in the top two quartiles at baseline ${ }^{22} 24$ and has shown an improvement in liver, adipose and peripheral tissue insulin sensitivity, ${ }^{24}$ making it a potentially attractive therapeutic agent for NASH. Recently, a phase IIb randomized double-blind placebo controlled trial (RDBPCT), GOLDEN-505, assessed the 
Table 1 Clinical trials of medications for non-alcoholic fatty liver disease (NAFLD)

\begin{tabular}{|c|c|c|c|c|c|}
\hline Medication & Mechanism & Current status & $\begin{array}{l}\text { Histological } \\
\text { benefit shown }\end{array}$ & $\begin{array}{l}\text { Studied in } \\
\text { cirrhotics }\end{array}$ & $\begin{array}{l}\text { Studied in diabetics } \\
\text { with NAFLD }\end{array}$ \\
\hline \multicolumn{6}{|c|}{ Medications with a primary metabolic target } \\
\hline Pioglitazone & PPAR $\gamma$ agonist & Phase III concluded* & Yes (secondary analyses only) & No & No \\
\hline Elafibranor & PPAR $\alpha / \delta$ agonist & Phase III & Yes (secondary analyses only) & No & Yes \\
\hline Saroglitazar & PPAR $\alpha / \gamma$ agonist & Phase III* & Pending $†$ & Yes & Yes \\
\hline Obeticholic acid & FXR agonist & Phase III* & Yes & No & Yes \\
\hline Liraglutide & GLP-1 receptor agonist & Phase II concluded* & Yes & Yes & Yes \\
\hline Aramchol & SCD inhibitor & Phase llb & Pending $†$ & No & Yes \\
\hline Volixibat (SHP-626) & ASBT inhibitor & Phase II & Pendingt & No & Yes \\
\hline BMS-986036 & FGF-21 analogue & Phase II & Not studied & $?$ & Yes \\
\hline NGM-282 & FGF-19 analogue & Phase II & Not studied & $?$ & $?$ \\
\hline Tesamorelin & GHRH analogue & Phase II* (HIV patients) & Pending $†$ & No & Yes \\
\hline NDI-010976 & ACC inhibitor & Phase I concluded & Not studied & No & No \\
\hline GS-9674 & FXR agonist & Phase I & Not studied & No & No \\
\hline Dur-928 & Sulfated oxysterol & Phase I & Not studied & Yes & Yes \\
\hline AZD4076 & miR-103/107 antagonist & Phase I & Not studied & No & No \\
\hline Rosuvastatin & HMG-CoA reductase inhibitor & Uncontrolled pilot concluded* & Yes & No & No \\
\hline INT-767 & FXR/TGR5 agonist & Preclinical & & & \\
\hline Sevelamer & Bile acid sequestrant & Preclinical* $^{*}$ & & & \\
\hline \multicolumn{6}{|c|}{ Medications targeting oxidative stress and inflammation } \\
\hline Vitamin E & Antioxidant & Phase III & Yes & No & No \\
\hline Pentoxifylline & PDE inhibitor & Phase II concluded & Yes & No & Yes \\
\hline Cenicriviroc & CCR2/CCR5 antagonist & Phase llb & Pending $\dagger$ & No & Yes \\
\hline Emricasan & Caspase inhibitors & Phase Ilb & Pendingt & No & Yes \\
\hline GS-4997 & ASK1 inhibitor & Phase II & Pendingt & No & Yes \\
\hline Amlexanox & IKKE/TBK1 inhibitor & Phase II* & Not studied & No & Yes \\
\hline PXS-4728A & VAP-1 inhibitor & Phase I concluded & Not studied & No & No \\
\hline \multicolumn{6}{|l|}{ Medications targeting the gut } \\
\hline Orlistat & Intestinal lipase inhibitor & Phase II concluded* & Inconclusive & Yes & Yes \\
\hline IMM-124e & IgG-rich bovine colostrum & Phase II & Not studied & No & Yes \\
\hline Solithromycin & Antibiotic & Phase II & Pending $†$ & No & Yes \\
\hline Faecal microbial transplant & Modulation of gut microbiome & Pilot & Not studied & No & Yes \\
\hline \multicolumn{6}{|l|}{ Antifibrotics } \\
\hline Simtuzumab & LOXL2 antibody & Phase II & Pending§ & Yes & Yes \\
\hline GR-MD-02 & Galectin-3 inhibitor & Phase II & Pending§ & Yes & Yes \\
\hline
\end{tabular}

*Currently approved for non-NAFLD indication.

tHistological outcome is studied in an ongoing trial.

¥Currently available over the counter.

§Histological and portal pressure outcomes are studied in an ongoing trial.

ACC, acetyl-CoA carboxylase; ASBT, apical sodium-dependent bile acid transporter; GLP, glucagon-like peptide; FGF, fibroblast growth factor; FXR, farnesoid X receptor; GHRH, growth hormone-releasing hormone; HMG-CoA, 3-hydroxy-3-methyl-glutaryl-coenzyme A; LOXL2, lysyl oxidase-like; PDE, phosphodiesterase; PPAR, peroxisome proliferator-activator receptor;

$\mathrm{SCD}$, stearoyl CoA desaturase; VAP, vascular adhesion protein.

effectiveness of elafibranor (80 or $120 \mathrm{mg} /$ day) or placebo for 1 year to treat biopsy-proven NASH. ${ }^{25}$ The study included 276 patients with mild-severe NASH and allowed for inclusion of diabetics, but excluded patients with cirrhosis. The primary end point of the study was ambitiously selected as histological resolution of NASH without worsening of fibrosis, based on recommendations from a recent US Food and Drug Administration/ American Association for the Study of Liver Diseases (AASLD) workshop. ${ }^{26}$ The primary end point was achieved in $23 \%$ and $21 \%$ of patients in the $80 \mathrm{mg}$ and $120 \mathrm{mg} /$ day groups, respectively, and in $17 \%$ of controls; the difference between the groups was not statistically significant. A more stringent definition of NASH resolution was assessed post hoc, and using that criteria, NASH resolution was achieved in $19 \%$ of the $120 \mathrm{mg} /$ day group compared with $12 \%$ of placebo-treated subjects $(p=0.045)$. The failure to show benefit appears to be primarily due to a high response rate in the placebo groups of mild-moderate (NAFLD activity score (NAS) ${ }^{27} 3-5$ ) disease and in fact, when subjects with mild disease at baseline $(\mathrm{NAS}=3)$ were excluded from the analysis, the $120 \mathrm{mg} /$ day dose was significantly superior than placebo across both response definitions. The $120 \mathrm{mg} /$ day dose had a modest effect on alanine aminotransferase (ALT) (decrease of $9.5 \mathrm{U} / \mathrm{L}$ compared with placebo) and in patients with diabetes improved insulin sensitivity. A phase III trial (NCT02704403) is currently recruiting subjects with $\mathrm{NASH}$ and $\mathrm{NAS} \geq 4$, aiming to determine the effects of 72 weeks of treatment with $120 \mathrm{mg} /$ day on NASH resolution without worsening of fibrosis. For the first time in therapeutic trials of NAFLD, a clinical co-primary end point is included, assessing the effect of elafibranor on mortality, cirrhosis and liver-related clinical outcomes.

$\operatorname{PPAR} \gamma$, another member of the PPAR family, is predominantly expressed in the adipose tissue, controlling lipogenesis, glucose metabolism and adipose tissue differentiation. Thiazolidinediones (TZDs), synthetic PPAR $\gamma$ agonists, are insulin sensitisers with proven efficacy for treatment of diabetes and have been shown in 
multiple trials to be effective for the treatment of NASH. ${ }^{28-30}$ In the PIVENS RDBPCT, the largest trial of a PPAR $\gamma$ agonist to date, 80 patients with NASH but not diabetes or cirrhosis received pioglitazone $30 \mathrm{mg} /$ day for 96 weeks and were compared with 83 placebo-treated subjects. ${ }^{30}$ Pioglitazone treatment was associated with histological improvement in 34\% of subjects compared with $19 \%$ of controls. The significance level of $\mathrm{p}=0.04$ did not meet the prespecified cut-off, despite apparent effectiveness, mainly due to discrepancies in the interpretation of entry liver biopsies in this group. Unfortunately, concerns with the safety profile of TZDs (especially regarding cardiovascular safety of rosiglitazone) and a side effect profile that includes weight gain due to redistribution of body fat have led to poor acceptance of these agents for the treatment of NASH in clinical practice. ${ }^{31}$

The glitazars are a class of medications designed as dual PPAR $\alpha / \gamma$ agonists, aiming to synergise the beneficial effects of PPAR $\alpha$ and PPAR $\gamma$ agonism. However, development of most compounds in this class has been halted due to safety concerns. The only glitazar in clinical use, saroglitazar, is currently approved in India for the treatment of diabetic dyslipidemia. ${ }^{32} 33$ In a mouse model of NASH, treatment with saroglitazar induced histological improvement as well as a decrease in liver fat content and ALT. $^{34}$ A retrospective analysis of patients with NAFLD treated with saroglitazar for dyslipidemia demonstrated significant and marked decrease in ALT (from $64 \pm 6$ to $28 \pm 3$, $\mathrm{p}<0.01$ ) after 24 weeks of treatment. ${ }^{35}$ In PRESS VIII, a phase II open-label study, the efficacy of saroglitazar was evaluated in 32 patients with biopsy-proven NASH and a $52 \%$ reduction in ALT was demonstrated after 12 weeks of treatment. ${ }^{36}$ A phase III RDBPCT of saroglitazar for 52 weeks in non-cirrhotic patients with biopsy-proven NASH is currently ongoing in India, with the primary end point defined as improvement in NASH histology with no worsening of fibrosis (Clinical Trials Registry-India CTRI/2015/10/006236).

Novel selective modulators of $\operatorname{PPAR} \alpha$ (pemafibrate, K-877) and PPAR $\gamma$ (INT-131), a PPAR $\delta$ agonist (HPP-593) and a PPAR $\alpha /$ $\gamma$ agonist (DSP-8658), are currently in clinical trials for other indications (mainly diabetic dyslipidemia). Whether these agents will prove to have a beneficial effect on NAFLD is yet unknown.

Although TZDs are typically considered to act through PPAR $\gamma$ agonism, there is evidence to suggest other mechanisms as well. In an animal model, MSDC-0602, an experimental TZD with poor affinity to $\operatorname{PPAR} \gamma$, was able to suppress hepatic glucose production and de novo lipogenesis in mice with hepatocyte-specific PPAR $\gamma$ knockout, ${ }^{37}$ likely through interaction with mitochondrial proteins Mcp1 and Mcp2. ${ }^{38}$ Clinical trials in human NASH are planned but have not begun to date.

\section{Targeting the FXR-bile acid axis}

The interaction of bile acids with the farnesoid $\mathrm{X}$ receptor (FXR), their intracellular receptor, negatively regulates bile acid synthesis and acts transcriptionally to decrease hepatic lipogenesis and steatosis, ${ }^{39}$ as well as decrease hepatic gluconeogenesis and improve peripheral insulin sensitivity. ${ }^{40}$ Obeticholic acid (6-ethylchenodeoxycholic acid (OCA)) is a synthetic lipophilic bile acid acting as FXR agonist and was recently evaluated as potential treatment for NASH in a phase IIb multicentre clinical trial. In the RDBPCT FLINT study, ${ }^{41} 283$ non-cirrhotic patients with biopsy-proven NASH (NAS $\geq 4$ ) were randomised to receive OCA ( $25 \mathrm{mg} /$ day) or placebo for 72 weeks. The primary end point of the study was histological improvement, defined as a decrease of two points in the NAS with no worsening of fibrosis. Histological improvement was seen in $45 \%$ of patients treated with OCA compared with $21 \%$ of those treated with placebo $(p=0.0002)$. Resolution of NASH was seen in $22 \%$ of patients versus $13 \%$ of controls $(p=0.08)$ and improvement in fibrosis score was detected in $35 \%$ versus $19 \%$ in controls $(p=0.004)$. A concomitant significant decrease in liver enzymes was noted. Interestingly, patients treated with OCA had a significant decrease in body mass index (BMI) (decrease of 0.7 vs gain of $0.1 \mathrm{~kg} / \mathrm{m}^{2}$ in the placebo group, $\left.\mathrm{p}=0.01\right)$; whether this was responsible, at least in part, for the histological improvement is unclear. However, patients treated with OCA had a reversible significant increase in total and low density lipoprotein (LDL)-cholesterol and decrease in HDL-cholesterol. These changes were of low magnitude, appeared predominantly at treatment initiation and improved with treatment continuation, suggesting adaptation or initiation of cholesterol-reducing medications. Whether these changes will be sustained with prolonged treatment and whether they will be associated with an increase in cardiovascular risk remains to be shown. The main side effect of OCA was pruritus, noted in 23\% of OCA-treated patients and requiring intervention or treatment discontinuation in several patients. Thus, although OCA appears effective for the treatment of $\mathrm{NASH}$, its long-term safety and tolerability is still unclear. A phase III trial (NCT02548351) is currently recruiting patients with non-cirrhotic NASH and will compare the effects of OCA or placebo for 72 weeks on two co-primary histological outcomes-resolution of NASH without worsening of fibrosis or improvement of fibrosis without worsening of $\mathrm{NASH}$. Similar to the elafibranor phase III trial, a clinical end point is included to assess the effect of treatment on mortality and liverrelated outcomes at 6 years.

Other FXR agonists are being tested in clinical trials. GS-9674, a synthetic non-steroidal FXR agonist, is currently in a phase I study. In contrast to OCA, GS-9674 and similar agents are less likely to undergo enterohepatic circulation and may have more predictable pharmacokinetics. ${ }^{42}$ INT-767 is a bile acid analogue that acts as a dual agonist on FXR and on TGR5, the transmembrane G-protein bile acid receptor. In an animal model, INT-767 improved histological features of NASH and modulated the activation of hepatic monocytes. ${ }^{43}$ A phase I trial in human subjects is scheduled to start. In contrast to OCA, treatment with ursodeoxycholic acid (UDCA), a naturally occurring secondary bile acid, has not been shown to improve histological features of NASH, ${ }^{44} 45$ despite lowering liver enzymes. The difference between the effects of UDCA and OCA may be related to the poor affinity of UDCA to FXR or even its ability to antagonise FXR activity. ${ }^{46}$ Other UDCA derivatives, such as nor-UDCA or tauroursodeoxycholic acid, were not tested in humans for the treatment of NAFLD.

An alternative approach to direct targeting of FXR has been the use of bile acid sequestrants that disrupt the enterohepatic circulation and result in reduction in serum lipids. An RDBPCT assessing the effectiveness of 24 weeks of colesevelam treatment in patients with biopsy-proven $\mathrm{NASH}^{47}$ failed to show histological improvement and in fact demonstrated a rise in liver enzymes and liver fat content with colesevelam treatment. These findings are consistent with a reduction in FXR and liver $\mathrm{X}$ receptor $(\mathrm{LXR})^{48}$ activation, as seen in animal models as well. ${ }^{48}$ All sequestrants may not be the same, as sevelamer, a phosphate-binding medication with bile salt binding capacity, ${ }^{49}$ has been shown in an animal model of steatohepatitis to improve liver fat, inflammation and fibrosis ${ }^{50}$ in an FXR-independent effect. ${ }^{51}$ Similarly to the effect of sequestration, enterohepatic circulation of bile acids can be disrupted by inhibiting the ileal apical sodium-dependent bile acid transporter (ASBT), the major route of reabsorption of bile acids in 
the terminal ileum. ASBT inhibitors cause an increase in faecal bile acids and improve glycaemic control in animal models. ${ }^{52}$ An oral inhibitor of ASBT, volixibat (SHP-626), recently concluded phase I studies and a phase II trial in patients with NASH is enrolling (NCT02787304).

\section{Inhibition of de novo lipogenesis}

Aramchol is a conjugate of cholic and arachidic acid that was shown to inhibit stearoyl CoA desaturase (SCD) and de novo lipogenesis in cell and animal models. ${ }^{53}$ In a recent phase II RDBPCT, $300 \mathrm{mg} /$ day of aramchol given for 3 months significantly decreased hepatic fat content by $12.5 \% .^{55}$ The study enrolled predominantly subjects with NAFLD but not NASH and did not use histological end points. Treatment was not associated with improvement in liver enzymes, raising a concern that the reduction in hepatic fat was not accompanied by an improvement in inflammation or cellular injury. A phase IIb study (NCT02279524) is currently evaluating the effects of higher doses (400 and $600 \mathrm{mg} /$ day) for 1 year in patients with non-cirrhotic biopsy-proven NASH (NAS $\geq 4$ ). The primary end point is decrease in liver fat content by MRI, while histological end points such as improvement or resolution of $\mathrm{NASH}$ are defined as secondary end points.

Malonyl-CoA acts as a key gatekeeper of fatty acid metabolism, controlling the balance between de novo lipogenesis and fatty acid oxidation. ${ }^{56}$ It serves as the building block for fatty acid synthesis and elongation, while inhibiting the entry of fatty acids to the mitochondria for $\beta$-oxidation. Acetyl-CoA carboxylase (ACC) is the key enzyme generating malonyl-CoA from acetyl-CoA and regulating this process. Inhibition of ACC by antisense oligonucleotides in a murine model of NAFLD increases fatty acid oxidation, decreases lipogenesis, decreased hepatic fat content and improved insulin sensitivity. ${ }^{56}$ An allosteric inhibitor of ACC, NDI-010976, was recently tested in obese subjects in a phase I trial ${ }^{57}$ and demonstrated dosedependent inhibition of de novo lipogenesis, reaching up to 98\% decrease from baseline following a single dose, making it a potential treatment for NAFLD.

Dur-928 is an endogenous sulfated oxysterol that has been shown to decrease hepatic fat content in animal models via inhibition of LXR and SREBP ${ }^{58}$ and is being developed as an oral agent for the treatment of NASH. A phase Ib study (ACTRN12615001355561) to evaluate its safety in patients with NASH compared with controls is currently ongoing.

\section{Incretins and DPP-4 inhibitors}

Glucagon-like peptide 1 (GLP-1) is an incretin hormone derived from the proglucagon polyprotein that is also the precursor to glucagon. ${ }^{59}$ GLP-1 is secreted by intestinal L-cells in response to meal ingestion and acts on the pancreas to improve glycaemic control by stimulating insulin secretion from pancreatic $\beta$-cells and inhibiting $\alpha$-cell glucagon secretion. Beyond the pancreas, GLP-1 improves peripheral insulin sensitivity, increases hepatic glucose uptake and glycogen synthesis, delays gastric emptying and decreases appetite. ${ }^{60}$ Several long-acting GLP-1 receptor agonists (GLP-1RAs) are approved for the treatment of type 2 diabetes mellitus. In retrospective analyses of GLP-1RA trials in diabetic subjects, beneficial effects on liver enzymes and hepatic fat content were shown. For example, liraglutide treatment for 26 weeks was associated with an average decrease of $8.2 \mathrm{U} / \mathrm{L}$ in ALT activity in subjects with baseline elevated ALT, but this appeared to be fully explained by the concomitant decrease in weight and HbA1c. ${ }^{61}$ Similar findings were reported for exenatide $^{62}$ and lixisenatide. ${ }^{62}$ Recently, the LEAN study ${ }^{63}$ was an
RDBPCT specifically designed to examine the utility of liraglutide to treat NASH. Fifty-two subjects with histologically proven NASH (only $33 \%$ of whom were diabetics) were randomised to receive liraglutide $(1.8 \mathrm{mg} /$ day $)$ or placebo for 48 weeks. At the end of the study, histological resolution of NASH without worsening of fibrosis, the primary end point, was seen in $39 \%$ of the patients assigned to liraglutide versus $9 \%$ of the placebo group $(p=0.02)$. Mechanistically, liraglutide treatment improved hepatic insulin sensitivity, with resultant reduction in hepatic endogenous glucose production, decreased hepatic de novo lipogenesis and promoted an improvement in adipose tissue insulin sensitivity with reduction of lipolysis and delivery of FFAs to the liver. ${ }^{64}$ As expected, treatment with liraglutide was associated with weight loss; histological responders lost on average $2.1 \mathrm{~kg}$ more than non-responders. Unfortunately, the study was not powered to show whether the beneficial effect on the liver was independent from the effect of weight loss alone.

A potentially alternative approach for augmentation of endogenous incretin effects is by use of small-molecule inhibitors of dipeptidyl peptidase 4 (DPP-4), the enzyme responsible for rapid degradation of GLP-1. Studies of sitagliptin, a DPP-4 inhibitor, were small and limited to diabetic patients with fatty liver. Only one study used histological end points. ${ }^{65}$ In this small, uncontrolled trial with 15 patients, treatment with $100 \mathrm{mg} /$ day of sitagliptin for 1 year was associated with improvement in liver enzymes, hepatocyte ballooning, histological activity scores and steatosis. A modest decrease in liver fat content by magnetic resonance spectroscopy (MRS) was also shown after 24 weeks of sitagliptin ${ }^{66}$ or vildagliptin. ${ }^{66}$ Other studies, on the other hand, failed to show an effect of sitagliptin treatment on liver fat content ${ }^{67}$ or liver enzymes. ${ }^{68}$ Recently, Cui et $a l^{69}$ performed the largest study to date, enrolling 50 subjects with NAFLD and pre-diabetes or early diabetes in an RDBPCT. In that study, 24 weeks of treatment with sitagliptin $100 \mathrm{mg} /$ day were not associated with improvement in liver fat, liver enzymes or liver stiffness. Thus, DPP-4 inhibition does not seem to be highly effective for the treatment of NASH.

\section{Lipid-lowering agents}

As metabolic syndrome is closely associated with fatty liver disease, many of the patients have dyslipidemia and increased risk for cardiovascular disease. Furthermore, in patients with $\mathrm{NASH}$, there is evidence for excess accumulation of free cholesterol in the liver, which could play a role in disease pathogenesis. $^{70} 71$ Statin use is generally safe in patients with chronic liver disease, including those with NAFLD. ${ }^{72}$ In a retrospective analysis, statin use was associated with decreased risk of NASH and advanced fibrosis in a large cohort of patients who underwent a liver biopsy for possible NASH. ${ }^{73}$ However, this could also reflect hesitancy of providers to prescribe statins to patients with advanced liver disease and in fact there is clear evidence for underuse of statins in patients with NAFLD. ${ }^{74}$ Prospective clinical trials using statins to treat the liver disease are few and limited but generally demonstrated a beneficial effect of statins on liver fat content (reviewed in ref. $^{72}$ ). In a recent prospective uncontrolled trial, 20 patients with biopsy-proven NASH (baseline NAS score of 8 ) and dyslipidemia were treated for 12 months with $10 \mathrm{mg} /$ day of rosuvastatin. In total, 19 of the 20 patients reportedly had a complete resolution of NASH including complete resolution of steatosis, inflammation and ballooning, despite experiencing no weight loss. ${ }^{75}$ Whether such phenomenal findings can be replicated in a larger controlled trial remains to be seen. 
In a pilot RDBPCT, ${ }^{76}$ ezetimibe, an inhibitor of intestinal absorption of cholesterol, was given for 24 weeks to patients with biopsy-proven NASH. Treatment had no effect on liver fat content, histology or liver enzymes.

\section{Targeting hormonal signalling}

FGF-21 is a peptide hormone, secreted predominantly from the liver. FGF-21 levels increase in the fasting state and regulate the fasting response in the adipose tissue and other organs. ${ }^{77}$ BMS-986036 (previously ARX-618) is a pegylated FGF-21 analogue that in animal models improved insulin sensitivity, hepatic fat content and de novo lipogenesis. ${ }^{78}$ A phase II RDBPCT is evaluating the effectiveness of 16 weeks of BMS-986036 treatment on hepatic fat content (by MRS) in patients with $\mathrm{NASH}$ (NCT02413372).

FXR activation in the terminal ileum by reabsorbed bile acids results in transcription and secretion of FGF-19, a peptide hormone that acts on the liver and leads to decreased bile acid synthesis and decreased gluconeogenesis in an insulin independent manner. ${ }^{77}$ It also has a proliferative effect on hepatocytes, predominantly through activation of the FGFR4 receptor, raising concern for potential tumourigenesis. ${ }^{79}$ NGM-282 is a recombinant variant FGF-19 that reportedly retains its beneficial metabolic effect but not the tumorigenic effects. In a mouse model of NASH, treatment with NGM-282 for 3 weeks resulted in a marked reduction of hepatic fat, histological features of NASH and decrease in $\mathrm{ALT}^{80}{ }^{0}$ NGM-282 is currently in a phase II study (NCT02443116) to determine the effects of 12 weeks of treatment on liver fat content in patients with biopsy-proven NASH.

VK2809 (previously MB07811) is a liver-directed agonist of the thyroid $\beta$ receptor. In murine models, VK2809 was shown to increase fatty acid oxidation and decrease hepatic fat content and plasma triglycerides. ${ }^{81} \mathrm{~A}$ phase II trial in patients with NAFLD and hypercholesterolaemia is scheduled to begin enrolling soon.

Tesamorelin, a growth hormone-releasing hormone (GHRH) analogue, is approved for treating HIV-associated lipodystrophy. In an RDBPCT on subjects with HIV and increased abdominal fat, tesamorelin treatment was associated with a mild decrease of $2 \%$ in liver fat content (by MRS) but not with any change in liver enzymes. ${ }^{82}$ However, given that baseline liver fat content and liver enzymes were normal or near-normal for many of the subjects, a specific effect on NAFLD could not be determined. A prospective trial in HIV-infected subjects with fatty liver and with histological and radiological end points is underway (NCT02196831). Although relative growth hormone (GH) deficiency has been associated with NAFLD and GH treatment may improve $\mathrm{NASH},{ }^{83}$ no studies in non-HIV NAFLD with tesamorelin or other $\mathrm{GH}$-axis agents have been conducted so far.

\section{MicroRNA-based treatment}

The microRNAs miR-103 and miR-107 are upregulated in the liver of obese animals ${ }^{84}$ and in the serum of human NAFLD patients. ${ }^{85}$ In animal models, miR-103/7 have been shown to modulate insulin sensitivity through direct interaction with caveolin-1. ${ }^{84}$ RG-125/AZD4076, an anti-Mir directed against miR-103/107 is being developed for the treatment of NASH and is currently in phase I (NCT02612662).

\section{MEDICATIONS AFFECTING OXIDATIVE STRESS AND INFLAMMATION Antioxidants}

Vitamin E, a fat-soluble antioxidant, has been used in multiple studies to treat fatty liver disease (table 1). In the PIVENS phase
III RDBPCT, ${ }^{30}$ vitamin $\mathrm{E}$ at a dose of $800 \mathrm{IU} /$ day for 96 weeks was superior to placebo, achieving histological response in $43 \%$ of subjects (compared with $19 \%$ of placebo, $\mathrm{p}=0.001$ ) and resolution of NASH in 36\%. Vitamin E treatment resulted in reduction in hepatocyte ballooning and lobular inflammation in approximately half of the patients, reflecting its expected effect as an antioxidant decreasing oxidative stress-mediated injury. Interestingly, it was also associated with a decrease in hepatic steatosis scores in 54\% of subjects, with an average decline of 0.7 units in the steatosis score, through an unclear mechanism. No effect on fibrosis was seen. The effects of vitamin $E$ were further demonstrated in a recent analysis ${ }^{86}$ that pooled together data from the PIVENS study and from patients receiving vitamin $\mathrm{E}$ on the placebo arm of the FLINT trial. ${ }^{41}$

Vitamin $\mathrm{E}$ is a mixture of eight different tocopherols and tocotrienols, with $\alpha$-tocopherol thought to be the main active component in humans due to its greater affinity to tocopherol transfer protein, the main transporter of vitamin $\mathrm{E}$ from the gut to the liver. ${ }^{87}$ Within the $\alpha$-tocopherol molecule, there are three chiral centres with eight possible enantiomers. The synthetic form of vitamin is a racemic mixture of different enantiomers and is approximately $50 \%$ as potent as the natural form, RRR- $\alpha$-tocopherol. Vitamin E formulations (considered a food supplement) markedly differ in their components and their racemic structure. Thus, when comparing results from different clinical trials or prescribing vitamin E treatment to patients, the specific formulation and doses need to be clarified. Currently, the best available data for the benefit of vitamin $\mathrm{E}$ in NASH, as discussed above, come from the PIVENS trial, ${ }^{30}$ which used natural, RRR- $\alpha$-tocopherol at a dose of $800 \mathrm{IU} /$ day.

Although vitamin $\mathrm{E}$ use was not associated with any major safety signals in clinical trials for NASH, data from other studies suggest it may not be a completely risk-free intervention as it is sometimes perceived. A large meta-analysis of 135000 patients from 19 studies that used vitamin E demonstrated an increase in all-cause mortality, ${ }^{88}$ although this has not been shown by other meta-analyses. ${ }^{89}$ Treatment with vitamin E was associated with an increased risk of haemorrhagic stroke, attributed to an antiplatelet effect of high-dose treatment. $^{90}$ In addition, the SELECT trial demonstrated an increased risk of prostate cancer in elderly men, receiving long-term vitamin $\mathrm{E}$ for cancer prevention. ${ }^{91}$ Although these studies suggest potential risks with vitamin $\mathrm{E}$ treatment, the overall magnitude of risk is relatively small and was seen in trials where the clinical benefit (for cancer prevention or prevention of cardiovascular disease) was low or non-existent. In the context of NASH, where the efficacy of vitamin $\mathrm{E}$ is well proven, these risks should be taken into consideration when selecting patients for treatment and compared against the risks of other possible medications or the risk of no treatment. Finally, the PIVENS trial excluded diabetics and patients with cirrhosis; thus, there is lack of data regarding vitamin E efficacy and safety in these patients.

Cysteamine is an aminothiol that can act as scavenger for reactive oxygen species and can replenish glutathione stores, and thus has potential benefit in NASH. In a small pilot study, Dohil et $a l^{67}$ treated 11 children with biopsy-proven NAFLD with enteric-coated cysteamine bitartarate for 24 weeks. There was a significant improvement in liver enzymes without an effect on BMI and an increase in serum adiponectin levels. Based on the results of this preliminary study, the NIDDK NASH-CRN conducted CyNCh, a phase IIb multicentre RDBPCT of cysteamine bitartarate. In total, 169 children with biopsy-proven NAFLD (NAS $\geq 4$ ) were randomised to weightbased cysteamine or placebo for 1 year. ${ }^{92}$ Although cysteamine 
therapy was associated with significant improvement in ALT (decrease of $53 \mathrm{U} / \mathrm{L}$ vs $8 \mathrm{U} / \mathrm{L}$ in controls, $\mathrm{p}=0.02$ ), there was no histological benefit, either in overall improvement of NASH (by NAS score) or in individual histological parameters.

\section{Targeting apoptosis and TNF $\alpha$ pathway}

A major component of steatohepatitis is hepatocyte injury and apoptosis, driven by both the intrinsic and extrinsic pathways, predominantly through tumour necrosis factor (TNF) $\alpha$ signalling. ${ }^{93}{ }^{94}$ Both pathways converge to a shared mechanism via the enzymatic cascade and activation of caspases, controlling apoptosis and inflammation. Emricasan is an oral irreversible pan-caspase inhibitor with high first-pass metabolism, ${ }^{95}$ making it a potentially attractive agent for the treatment of liver diseases associated with apoptosis. Emricasan has been shown to decrease liver enzymes in patients with chronic hepatitis $\mathrm{C}^{96} 97$ and to markedly improve inflammation, hepatocyte injury and fibrosis in mice fed a high-fat diet (HFD), without having an effect on hepatic fat accumulation or features of the metabolic syndrome. ${ }^{98}$ Recently, in a short-term phase II RDBPCT, 38 subjects with non-cirrhotic NAFLD and elevated transaminases were randomised to receive emricasan $25 \mathrm{mg}$ twice daily or placebo for 28 days. $^{99}$ The emricasan-treated group demonstrated marked decrease in liver enzymes (median ALT decrease 25.8 vs $9.4 \mathrm{U} / \mathrm{L}$ for placebo, $\mathrm{p}<0.05$ ) and, as expected, a decrease in serum cytokeratin 18 fragments, a marker of liver apoptosis. Whether this improvement in liver enzymes is associated with a histological improvement is yet to be seen. ENCORE-NF, an ongoing phase IIb trial (NCT02686762), is evaluating the efficacy of two doses of emricasan $(10 \mathrm{mg}$ and $100 \mathrm{mg} /$ day) for 72 weeks in patients with biopsy-proven NASH and fibrosis (but not cirrhosis). The primary end point is improvement in fibrosis without worsening of NASH, with secondary end points aiming to demonstrate histological improvement or resolution of NASH.

Pentoxifylline has been suggested as a potentially beneficial therapy for NASH due to its putative effects on TNFo, reduction of oxidative stress and possible antifibrotic effects. Furthermore, it was initially thought to be beneficial for the treatment of severe acute alcoholic hepatitis, ${ }^{100}$ although this has not been replicated in later, larger trials. ${ }^{101} 102$ In a small, two-centre RDBPCT, Zein et al ${ }^{103}$ treated 55 patients with biopsy-proven NASH with pentoxifylline $400 \mathrm{mg}$ three times daily (or placebo) for 1 year. Histological improvement, defined as a decrease in NAS by $\geq 2$ points, occurred in $38.5 \%$ of patients treated with pentoxifylline compared with $13.8 \%$ of placebo-treated ones $(p=0.036)$. The main factors driving the improvement were a reduction in steatosis and inflammation, while hepatocyte ballooning did not change significantly from baseline. There was also a modest $(0.2$ unit) but significant decrease in fibrosis scores. Resolution of NASH was seen in $25 \%$ of patients treated with pentoxifylline ( $p=0.03$ for the comparison with placebo). No effect was seen on markers of insulin sensitivity or on serum $\mathrm{TNF} \alpha$ levels. Histological response to pentoxifylline was associated with a decrease in plasma levels of several oxidised lipids, ${ }^{104}$ suggesting that a reduction in lipid peroxidation may underlie the beneficial effect. Two recent meta-analyses ${ }^{105} 106$ included that study as well as several other smaller, lower-quality ones and concluded that pentoxifylline has a beneficial effect on liver enzymes and histology.

Apoptosis signal-regulating kinase 1 (ASK1) is a MAP3 kinase that upon activation by extracellular TNF $\alpha$ or intracellular oxidative or endoplasmic reticulum stress activates the p38/JNK pathway, resulting in hepatocyte apoptosis and fibrosis. In murine models, it has also been shown to contribute to TNF $\alpha$-mediated insulin resistance and steatosis ${ }^{107}$ and inhibition of ASK1 ameliorates diet-induced steatosis and fibrosis. ${ }^{108}$ In an ongoing randomised open-label phase II trial, GS-4997, an oral ASK1 inhibitor, is studied for 24 weeks with or without simtuzumab in patients with NASH and stage 2-3 fibrosis (NCT 02466516).

\section{Immune modulators}

Two IкB kinases (IKKs)-IKKe and TBK1-appear to be important in linking obesity and inflammation. Both are upregulated in adipose tissue in animals with diet-induced obesity and deletion protects these animals from insulin resistance, obesity and steatosis. ${ }^{109}$ Amlexanox, a medication used to treat asthma and aphthous ulcers, was found in a screening assay to be an inhibitor of both IKKe and TBK1 and in animal models of diet-induced or genetic obesity caused weight loss through increased energy expenditure. ${ }^{110}$ In these animals, amlexanox treatment improved insulin sensitivity and decreased steatosis and hepatic expression of inflammatory genes. A phase II RDBPCT is currently assessing the effects of 12 weeks of amlexanox in patients with diabetes, obesity and fatty liver on hepatic fat content by imaging, HbA1c and weight (NCT01975935).

In NASH, there is overexpression of inflammatory chemokines, including CCL2 (MCP1) and CCL5 (RANTES), ${ }^{111}$ and these play an important role in the activation and migration of inflammatory cells into the liver, as well as the progression of fibrosis. ${ }^{112} 113$ Cenicriviroc is an oral antagonist of CCR2/ CCR5, the chemokine receptors for MCP1 and RANTES, respectively. In clinical trials of HIV-infected patients, cenicriviroc was associated with improvement in serum markers of fibrosis, although in patients with no apparent liver disease. ${ }^{114}$ Cenicriviroc is currently studied in patients with obesity, insulin resistance and suspected NAFLD in a phase IIa study (ORION) aiming to assess the effects of 24-week treatment on insulin sensitivity, liver enzymes and liver imaging. Simultaneously, CENTAUR, an ongoing phase IIb trial, is assessing the histological effects of up to 2 years of cenicriviroc or placebo on patients with NASH and fibrosis (but not cirrhosis). ${ }^{115}$ The results of both studies are expected later this year.

Vascular adhesion protein 1 (VAP-1, also known as SSAO) is a membrane sialoglycoprotein expressed on hepatic sinusoidal endothelial cells. VAP-1 functions as a receptor to support adhesion and recruitment of lymphocytes to the liver. ${ }^{116}$ VAP-1 also has amine oxidase activity, which can result in the generation of reactive oxygen species and subsequent injury. In patients with NAFLD, serum levels of the soluble form of VAP-1 are increased and hepatic VAP-1 expression is upregulated; furthermore, VAP-1 is also detected in these patients on activated stellate cells, the major mediators of fibrosis. ${ }^{117}$ Inhibition or knockdown of VAP-1 decreased hepatic inflammation, injury and fibrosis in murine models of NASH. ${ }^{117}$ PXS-4728A, a selective irreversible inhibitor of the VAP-1 enzymatic activity, improved histological features of NASH in the streptozotocin (STZ)/HFD mouse model and completed phase I in healthy volunteers. ${ }^{118}$

\section{MEDICATIONS WITH A PRIMARY GUT TARGET Antiobesity medications}

Lifestyle modification has been consistently shown to improve fatty liver disease ${ }^{119} 120$ and should be considered the backbone of any therapeutic intervention (table 1). ${ }^{121}$ However, sustained (as opposed to short term) weight loss is difficult to achieve and maintain. Orlistat is a gut lipase inhibitor, decreasing the absorption of dietary fats and approved for the treatment of obesity. In 
a small prospective RDBPCT, orlistat treatment for 6 months was associated with a significant decrease in ALT and liver fat by ultrasound compared with the control, diet-alone, group, despite a similar degree of weight loss. ${ }^{122}$ However, in another prospective open-label study, 9 months of orlistat, vitamin E and diet were compared with vitamin $\mathrm{E}$ and diet alone. Weight loss in both groups was associated with histological improvement as well as decrease in liver enzymes, but there was no difference between the orlistat-treated subjects and controls. ${ }^{123}$ Interestingly, there was no significant difference in the degree of weight loss between the subjects treated with orlistat and controls, but this may reflect the small sample size and lack of sufficient power. Furthermore, since vitamin E (which was subsequently shown to have beneficial effects on NAFLD) was included in both treatment groups, and since it is fat-soluble, impaired absorption of the vitamin E supplementation in the orlistat group could have offset the benefits from this intervention. Thus, although orlistat is not likely to provide benefit independently of weight loss, it can be considered as an adjunct to assist in weight loss in patients with NAFLD.

\section{Targeting gut microbiome}

The gut microbiome is involved in the pathogenesis of NAFLD and $\mathrm{NASH},{ }^{124}$ at least in part through exposure of the liver to bacterial products such as lipopolysaccharide (LPS). IMM-124e is an IgG-rich extract of bovine colostrum obtained from cows immunised against LPS. In ob/ob mice, oral IMM-124e treatment is associated with improved liver fat content, liver enzymes and insulin sensitivity, ${ }^{125}$ thought to be due to a reduction of delivery of LPS and bacterial products from the gut to the liver and subsequent activation of Kupffer cells. In a small pilot study on 10 patients with biopsy-proven NASH, 30 days of IMM-124e treatment improved insulin sensitivity and glycaemic control, with a small effect on liver enzymes. ${ }^{126}$ A phase II RDBPCT is currently evaluating the effects of 24 weeks of IMM-124e in patients with biopsy-proven NASH (NCT02316717). The study end points include changes in liver fat content (by MRS) and in liver enzymes.

The effects of the gut on the liver can also be addressed by modulating the gut microbiome itself, through a faecal microbiota transplantation (FMT). FMT is a proven effective therapy for recurrent Clostridium difficile infection and transplantation of gut microbiota from lean subjects was shown to improve insulin sensitivity in subjects with the metabolic syndrome. ${ }^{127}$ In an ongoing open-label pilot study, investigators are performing FMT from lean donors to patients with biopsy-proven noncirrhotic NASH to assess the effects on liver fat content and markers of injury (NCT02469272). Although FMT is unlikely to become a main therapeutic option for NAFLD, the results of the study will hopefully shed light on an important element of the disease pathogenesis.

Solithromycin is a new generation macrolide antibiotic in clinical trials for the treatment of bacterial infections. In a murine model of diet and STZ-induced NASH, solithromycin has demonstrated reduction in hepatocyte ballooning and inflammation, without an effect on liver fat content ${ }^{128}$ as well as a decrease in blood glucose levels and downregulation of hepatic gluconeogenic enzymes. ${ }^{129}$ The mechanism of response may not be related to solithromycin's antibacterial activity as it is not active against gut Gram-negative bacteria. Based on the preclinical results, a phase II open-label study is currently ongoing to determine the effect of 13 weeks of solithromycin treatment on liver histology in patients with non-cirrhotic NASH (NCT02510599).

\section{ANTIFIBROTIC MEDICATIONS}

Given the clear association between hepatic fibrosis and clinical outcomes in liver disease in general and in NASH in particular, ${ }^{7}$ reduction in fibrosis is a main goal of treatment (table 1). Beyond targeting NASH-specific disease mechanisms, as detailed above, several medications focus on the fibrotic process itself in a NASH-independent manner. Simtuzumab is a monoclonal antibody against lysyl oxidase-like 2 (LOXL2), a matrix enzyme responsible for cross-linking of collagen chains that is expressed extensively in fibrotic regions in the liver. ${ }^{130}$ Simtuzumab is currently evaluated in a long-term phase II study (NCT01672866) to determine whether it can decrease hepatic collagen in noncirrhotic subjects with NASH and advanced fibrosis. In cirrhotic patients with NASH, simtuzumab treatment for 2 years is being studied (NCT01672879) with the goal of decreasing hepatic venous pressure gradient (HVPG), as well as a reduction in liver-related mortality, transplant or decompensation. Both studies have completed enrolment but results are not available so far.

Galectin-3 is a protein expressed predominantly in immune cells that recognises and binds to galactose residues. Galectin-3 is essential to the development of liver fibrotic process in NASH and GR-MD-02, a galectin-3 inhibitor, decreased $\mathrm{NASH}$ disease activity and fibrosis in an animal model. ${ }^{131}$ GR-MD-02 is currently evaluated in two phase II clinical trials; one trial is recruiting patients with $\mathrm{NASH}$ cirrhosis and portal HTN to evaluate the ability of 1-year treatment to reduce HVPG (NCT02462967), while the other study is evaluating the reduction of hepatic fibrosis by MRI after 16 weeks of treatment in patients with NASH and advanced fibrosis (NCT02421094).

\section{SUMMARY}

In recent years, remarkable progress has been made in understanding the pathogenesis of NAFLD/NASH and, subsequently, in developing medications to target the disease. Interestingly, despite this progress, histological improvement of NASH has not exceeded $50 \%$ and $\mathrm{NASH}$ resolution rates are remarkably lower. This may reflect ineffectiveness of therapies, inability to overcome continuing caloric excess, or the heterogeneity in pathway activation between subjects. It is possible that no one-medication-fits-all strategy will be successful and future research should assess predictors of response to specific therapies.

It is becoming apparent from clinical trials that the short-term results of therapeutic intervention will depend on the target for therapy. Agents aimed at metabolic targets are likely to impact steatosis first, and then inflammation and ballooning followed by fibrosis, whereas agents targeting the interface of inflammation and fibrosis or fibrosis alone will impact their target. While prevention of progression to cirrhosis is a critical end point and a marker of therapeutic benefit, it should be noted that therapies based simply on pure antifibrotic effects do not address the drivers of disease progression such as cell stress, apoptosis and inflammation. The long-term implications of impacting fibrosis without affecting disease activity remain to be determined but may set the stage for combination therapies along with drugs targeting the metabolic components of the disease. Conversely, the benefits of drugs targeting metabolic targets may be amplified by adding agents with anti-inflammatory and antifibrotic effects. The current understanding of the pathophysiology of $\mathrm{NASH}$ provides a strong rationale for combination therapeutics for NASH and it is hoped that current agents in pivotal trials 
will be used to achieve high rates of response in use as combination therapy.

Currently, no pharmacological therapy is approved for NAFLD or NASH. Clinicians treating these patients should emphasise the importance of lifestyle changes, weight loss and exercise as the mainstay of treatment. Orlistat can be safely used as an adjunct to weigh loss and may provide additional benefit. Clinicians should also address and treat other components of the metabolic syndrome, including hypertension, dyslipidemia and insulin resistance or diabetes. Statins can be safely used to treat hypercholesterolaemia in patients with NAFLD or NASH. For patients with biopsy-proven NASH, currently available medications with proven benefit, although for off-label use, include liraglutide, pioglitazone, vitamin $\mathrm{E}$ and pentoxifylline. It should be noted that the vast majority of trials excluded patients with cirrhosis and these are best treated in the context of clinical studies.

Given the breadth of the pipeline of new agents and its dynamicity, in the coming years the arsenal of medications available to the hepatologist treating NASH is likely to significantly expand.

Contributors YR: manuscript design, writing, revision and approval of final version. AJS: manuscript design, revision and approval of final version.

Funding NIDDK Intramural Research Program.

Competing interests YR is funded by the NIDDK intramural Research Program.

AJS has stock options in Genfit. He has served as a consultant to AbbVie, Astra Zeneca, Nitto Denko, Nimbus, Salix, Tobira, Takeda, Fibrogen, Immuron, Exhalenz and Genfit. He has been an unpaid consultant to Intercept and Echosens. His institution has received grant support from Gilead, Salix, Tobira and Novartis. None of these are related to the current study. He is the president and CMO of Sanyal Biotechnologies.

Provenance and peer review Commissioned; externally peer reviewed.

\section{REFERENCES}

1 Browning JD, Szczepaniak LS, Dobbins $R$, et al. Prevalence of hepatic steatosis in an urban population in the United States: impact of ethnicity. Hepatology 2004:40:1387-95.

2 Williams CD, Stengel J, Asike Ml, et al. Prevalence of nonalcoholic fatty liver disease and nonalcoholic steatohepatitis among a largely middle-aged population utilizing ultrasound and liver biopsy: a prospective study. Gastroenterology 2011;140:124-31.

3 Wong VW, Chu WC, Wong GL, et al. Prevalence of non-alcoholic fatty liver disease and advanced fibrosis in Hong Kong Chinese: a population study using proton-magnetic resonance spectroscopy and transient elastography. Gut 2012;61:409-15.

4 Kanwal F, Kramer JR, Duan Z, et al. Trends in the burden of nonalcoholic fatty liver disease in a United States cohort of veterans. Clin Gastroenterol Hepatol 2016:14:301-8.e1-2.

5 Younossi ZM, Stepanova M, Afendy $M$, et al. Changes in the prevalence of the most common causes of chronic liver diseases in the United States from 1988 to 2008. Clin Gastroenterol Hepatol 2011;9:524-30.e1; quiz e60.

6 Angulo P. Nonalcoholic fatty liver disease. N Engl J Med 2002;346:1221-31.

7 Ekstedt $\mathrm{M}$, Hagstrom $\mathrm{H}$, Nasr $\mathrm{P}$, et al. Fibrosis stage is the strongest predictor for disease-specific mortality in NAFLD after up to 33 years of follow-up. Hepatology 2015;61:1547-54.

8 Sanyal AJ, Campbell-Sargent C, Mirshahi F, et al. Nonalcoholic steatohepatitis: association of insulin resistance and mitochondrial abnormalities. Gastroenterology 2001;120:1183-92.

9 Donnelly KL, Smith Cl, Schwarzenberg SJ, et al. Sources of fatty acids stored in liver and secreted via lipoproteins in patients with nonalcoholic fatty liver disease. J Clin Invest 2005;115:1343-51.

10 Lambert JE, Ramos-Roman MA, Browning JD, et al. Increased de novo lipogenesis is a distinct characteristic of individuals with nonalcoholic fatty liver disease. Gastroenterology 2014;146:726-35.

11 Fabbrini E, Mohammed BS, Magkos F, et al. Alterations in adipose tissue and hepatic lipid kinetics in obese men and women with nonalcoholic fatty liver disease. Gastroenterology 2008;134:424-31.

12 Fabbrini E, Sullivan S, Klein S. Obesity and nonalcoholic fatty liver disease: biochemical, metabolic, and clinical implications. Hepatology 2010;51:679-89.
13 Neuschwander-Tetri BA. Hepatic lipotoxicity and the pathogenesis of nonalcoholic steatohepatitis: the central role of nontriglyceride fatty acid metabolites. Hepatology 2010;52:774-88.

14 Poulsen L, Siersbæk M, Mandrup S. PPARs: fatty acid sensors controlling metabolism. Semin Cell Dev Biol 2012;23:631-9.

15 Brown JD, Plutzky J. Peroxisome proliferator-activated receptors as transcriptional nodal points and therapeutic targets. Circulation 2007;115:518-33.

16 Kersten S, Seydoux J, Peters JM, et al. Peroxisome proliferator-activated receptor alpha mediates the adaptive response to fasting. I Clin Invest 1999;103:1489-98.

17 Montagner A, Polizzi A, Fouché E, et al. Liver PPAR $\alpha$ is crucial for whole-body fatty acid homeostasis and is protective against NAFLD. Gut 2016;65:1202-14.

18 Musso G, Gambino R, Cassader M, et al. A meta-analysis of randomized trials for the treatment of nonalcoholic fatty liver disease. Hepatology 2010;52:79-104.

19 Bojic LA, Huff MW. Peroxisome proliferator-activated receptor $\delta$ : a multifaceted metabolic player. Curr Opin Lipidol 2013;24:171-7.

20 Bojic LA, Burke AC, Chhoker SS, et al. Peroxisome proliferator-activated receptor $\delta$ agonist GW1516 attenuates diet-induced aortic inflammation, insulin resistance, and atherosclerosis in low-density lipoprotein receptor knockout mice. Arterioscler Thromb Vasc Biol 2014;34:52-60.

21 Risérus U, Sprecher $\mathrm{D}$, Johnson T, et al. Activation of peroxisome proliferator-activated receptor (PPAR)delta promotes reversal of multiple metabolic abnormalities, reduces oxidative stress, and increases fatty acid oxidation in moderately obese men. Diabetes 2008;57:332-9.

22 Staels B, Rubenstrunk A, Noel B, et al. Hepatoprotective effects of the dual peroxisome proliferator-activated receptor alpha/delta agonist, GFT505, in rodent models of nonalcoholic fatty liver disease/nonalcoholic steatohepatitis. Hepatology 2013;58:1941-52.

23 Hanf R, Millatt $\mathrm{L}$, Cariou B, et al. The dual peroxisome proliferator-activated receptor alpha/delta agonist GFT505 exerts anti-diabetic effects in $\mathrm{db} / \mathrm{db}$ mice without peroxisome proliferator-activated receptor gamma-associated adverse cardiac effects. Diab Vasc Dis Res 2014;11:440-7.

24 Cariou B, Hanf R, Lambert-Porcheron $\mathrm{S}$, et al. Dual peroxisome proliferator-activated receptor $\alpha / \delta$ agonist GFT505 improves hepatic and peripheral insulin sensitivity in abdominally obese subjects. Diabetes Care 2013;36:2923-30.

25 Ratziu V, Harrison S, Francque S, et al. Elafibranor, an agonist of the peroxisome proliferator-activated receptor- $\alpha$ and $-\delta$, induces resolution of nonalcoholic steatohepatitis without fibrosis worsening. Gastroenterology 2016;150:1147-59. e5.

26 Sanyal AJ, Friedman SL, McCullough AJ, et al. Challenges and opportunities in drug and biomarker development for nonalcoholic steatohepatitis: findings and recommendations from an American Association for the Study of Liver Diseases-U. S. Food and Drug Administration Joint Workshop. Hepatology 2015;61:1392-405.

27 Kleiner DE, Brunt EM, Van Natta M, et al. Design and validation of a histological scoring system for nonalcoholic fatty liver disease. Hepatology 2005;41:1313-21.

28 Belfort $R$, Harrison SA, Brown $K$, et al. A placebo-controlled trial of pioglitazone in subjects with nonalcoholic steatohepatitis. N Engl J Med 2006;355:2297-307.

29 Promrat K, Lutchman G, Uwaifo Gl, et al. A pilot study of pioglitazone treatment for nonalcoholic steatohepatitis. Hepatology 2004:39:188-96.

30 Sanyal AJ, Chalasani N, Kowdley KV, et al. Pioglitazone, vitamin E, or placebo for nonalcoholic steatohepatitis. N Engl J Med 2010;362:1675-85.

31 Rinella ME, Lominadze $Z$, Loomba R, et al. Practice patterns in NAFLD and NASH: real life differs from published guidelines. Therap Adv Gastroenterol 2016;9:4-12.

32 Pai V, Paneerselvam A, Mukhopadhyay S, et al. A multicenter, prospective, randomized, double-blind study to evaluate the safety and efficacy of Saroglitazar 2 and $4 \mathrm{mg}$ compared to Pioglitazone $45 \mathrm{mg}$ in diabetic dyslipidemia (PRESS V). J Diabetes Sci Technol 2014;8:132-41.

33 Jani RH, Pai V, Jha P, et al. A multicenter, prospective, randomized, double-blind study to evaluate the safety and efficacy of Saroglitazar 2 and $4 \mathrm{mg}$ compared with placebo in type 2 diabetes mellitus patients having hypertriglyceridemia not controlled with atorvastatin therapy (PRESS VI). Diabetes Technol Ther 2014;16:63-71.

34 Jain MR, Giri SR, Bhoi B, et al. Saroglitazar shows therapeutic benefits in mouse model of nonalcoholic fatty liver disease (NAFLD) and Nonalcoholic Steatohepatitis (NASH). Diabetes 2015;64:A503-A.

35 Saboo BD, Prajapati A, Joshi S, et al. To Assess the Effect of $4 \mathrm{mg}$ Saroglitazar on patients of diabetes dyslipidemia with nonalcoholic fatty liver disease for 24 weeks at diabetes care centre. Diabetes 2015;64:A180-A.

36 Giri S, Bhoi B, Trivedi C, et al. Efficacy of Saroglitazar, a Novel PPAR $\alpha / \gamma$ Agonist in a Mouse Model of Non-Alcoholic Steatohepatitis. Keystone Symposium - Liver Metabolism and Nonalcoholic Fatty Liver Disease (NAFLD). Whistler, BC, Canada, 2015.

37 Chen Z, Vigueira PA, Chambers KT, et al. Insulin resistance and metabolic derangements in obese mice are ameliorated by a novel peroxisome proliferator-activated receptor $\gamma$-sparing thiazolidinedione. J Biol Chem 2012;287:23537-48

38 Colca JR, McDonald WG, Cavey GS, et al. Identification of a mitochondrial target of thiazolidinedione insulin sensitizers (mTOT)--relationship to newly identified mitochondrial pyruvate carrier proteins. PloS ONE 2013;8:e61551. 
39 Watanabe $M$, Houten $S M$, Wang $L$, et al. Bile acids lower triglyceride levels via a pathway involving FXR, SHP, and SREBP-1C. J Clin Invest 2004;113:1408-18.

40 Porez G, Prawitt J, Gross B, et al. Bile acid receptors as targets for the treatment of dyslipidemia and cardiovascular disease. J Lipid Res 2012;53:1723-37.

41 Neuschwander-Tetri BA, Loomba R, Sanyal AJ, et al. Farnesoid X nuclear receptor ligand obeticholic acid for non-cirrhotic, non-alcoholic steatohepatitis (FLINT): a multicentre, randomised, placebo-controlled trial. Lancet 2015;385:956-65.

42 Gege C, Kinzel O, Steeneck C, et al. Knocking on FXR's door: the "hammerhead"-structure series of FXR agonists-amphiphilic isoxazoles with potent in vitro and in vivo activities. Curr Top Med Chem 2014;14:2143-58.

43 McMahan RH, Wang XX, Cheng LL, et al. Bile acid receptor activation modulates hepatic monocyte activity and improves nonalcoholic fatty liver disease. J Biol Chem 2013;288:11761-70.

44 Ratziu V. Treatment of NASH with ursodeoxycholic acid: pro. Clin Res Hepatol Gastroenterol 2012;36(Suppl 1):S41-5.

45 Liechti F, Dufour JF. Treatment of NASH with ursodeoxycholic acid: cons. Clin Res Hepatol Gastroenterol 2012;36(Suppl 1):S46-52.

46 Mueller M, Thorell A, Claudel T, et al. Ursodeoxycholic acid exerts farnesoid X receptor-antagonistic effects on bile acid and lipid metabolism in morbid obesity. J Hepatol 2015;62:1398-404.

47 Le TA, Chen J, Changchien $C$, et al. Effect of colesevelam on liver fat quantified by magnetic resonance in nonalcoholic steatohepatitis: a randomized controlled trial. Hepatology 2012;56:922-32.

48 Herrema $H$, Meissner $M$, van Dijk TH, et al. Bile salt sequestration induces hepatic de novo lipogenesis through farnesoid $X$ receptor- and liver $X$ receptor alpha-controlled metabolic pathways in mice. Hepatology 2010;51:806-16.

49 Braunlin W, Zhorov E, Guo A, et al. Bile acid binding to sevelamer $\mathrm{HCl}$. Kidney Int 2002:62:611-19.

50 McGettigan B, McMahan R, Porsche C, et al. Sevelamer improves hepatic steatosis and modulates inflammation in a Western Diet mouse model of non-alcoholic fatty liver disease (NAFLD). Hepatology 2014;60:746A-7A.

51 Luo Y, Wang XX, Orlicky DJ, et al. Bile Acid Sequestrant Prevents NAFLD/NASH in Diet Induced Obesity and Insulin Resistant Mice. Keystone Symposium - Liver Metabolism and Nonalcoholic Fatty Liver Disease (NAFLD). Whistler, BC, Canada, 2015.

52 Chen L, Yao X, Young A, et al. Inhibition of apical sodium-dependent bile acid transporter as a novel treatment for diabetes. Am J Physiol Endocrinol Metab 2012;302:E68-76

53 Leikin-Frenkel A, Gonen A, Shaish A, et al. Fatty acid bile acid conjugate inhibits hepatic stearoyl coenzyme A desaturase and is non-atherogenic. Arch Med Res 2010:41:397-404.

54 Leikin-Frenkel A, Goldiner I, Leikin-Gobbi D, et al. Treatment of preestablished diet-induced fatty liver by oral fatty acid-bile acid conjugates in rodents. Eur J Gastroenterol Hepatol 2008;20:1205-13.

55 Safadi R, Konikoff FM, Mahamid M, et al. The fatty acid-bile acid conjugate Aramchol reduces liver fat content in patients with nonalcoholic fatty liver disease. Clin Gastroenterol Hepatol 2014;12:2085-91.e1.

56 Foster DW. Malonyl-CoA: the regulator of fatty acid synthesis and oxidation. J Clin Invest 2012;122:1958-9.

57 Westlin WF, Blanchette H, Harriman G, et al. NDI-010976, a potent, liver-directed, oral inhibitor of acetyl coa carboxylase for non-alcoholic steatohepatitis: pharmacodynamic effects on hepatic de novo lipogenesis in obese but otherwise healthy adult male volunteers. J Hepatol 2016;64:S190-S1.

58 Ren S, Ning Y. Sulfation of 25-hydroxycholesterol regulates lipid metabolism, inflammatory responses, and cell proliferation. Am J Physiol Endocrinol Metab 2014;306:E123-30

59 Holst JJ. The physiology of glucagon-like peptide 1. Physiol Rev 2007:87:1409-39.

60 Abu-Hamdah R, Rabiee A, Meneilly GS, et al. Clinical review: The extrapancreatic effects of glucagon-like peptide- 1 and related peptides. J Clin Endocrinol Metab 2009;94:1843-52.

61 Armstrong MJ, Houlihan DD, Rowe IA, et al. Safety and efficacy of liraglutide in patients with type 2 diabetes and elevated liver enzymes: individual patient data meta-analysis of the LEAD program. Aliment Pharmacol Ther 2013;37:234-42.

62 Buse JB, Klonoff DC, Nielsen LL, et al. Metabolic effects of two years of exenatide treatment on diabetes, obesity, and hepatic biomarkers in patients with type 2 diabetes: an interim analysis of data from the open-label, uncontrolled extension of three double-blind, placebo-controlled trials. Clin Ther 2007:29:139-53.

63 Armstrong MJ, Gaunt P, Aithal GP, et al. Liraglutide safety and efficacy in patients with non-alcoholic steatohepatitis (LEAN): a multicentre, double-blind, randomised, placebo-controlled phase 2 study. Lancet 2016;387:679-90.

64 Armstrong MJ, Hull D, Guo K, et al. Glucagon-like peptide 1 decreases lipotoxicity in non-alcoholic steatohepatitis. J Hepatol 2016;64:399-408.

65 Yilmaz Y, Yonal O, Deyneli O, et al. Effects of sitagliptin in diabetic patients with nonalcoholic steatohepatitis. Acta Gastroenterol Belg 2012;75:240-4.

66 Kato H, Nagai Y, Ohta A, et al. Effect of sitagliptin on intrahepatic lipid content and body fat in patients with type 2 diabetes. Diabetes Res Clin Pract 2015;109:199-205.
67 Dohil R, Schmeltzer S, Cabrera BL, et al. Enteric-coated cysteamine for the treatment of paediatric non-alcoholic fatty liver disease. Aliment Pharmacol Ther 2011;33:1036-44.

68 Fukuhara $\mathrm{T}$, Hyogo $\mathrm{H}$, Ochi $\mathrm{H}$, et al. Efficacy and safety of sitagliptin for the treatment of nonalcoholic fatty liver disease with type 2 diabetes mellitus Hepatogastroenterology 2014;61:323-8.

69 Cui JY, Philo L, Nguyen P, et al. Sitagliptin versus placebo in the treatment of non-alcoholic fatty liver disease: a randomized controlled trial. J Hepatol 2016;64: S192-3.

70 Puri P, Baillie RA, Wiest MM, et al. A lipidomic analysis of nonalcoholic fatty liver disease. Hepatology 2007;46:1081-90.

71 Farrell GC, van Rooyen D. Liver cholesterol: is it playing possum in NASH? Am J Physiol Gastrointest Liver Physiol 2012;303:G9-11.

72 Pastori D, Polimeni L, Baratta F, et al. The efficacy and safety of statins for the treatment of non-alcoholic fatty liver disease. Dig Liver Dis 2015;47:4-11.

73 Dongiovanni P, Petta S, Mannisto V, et al. Statin use and non-alcoholic steatohepatitis in at risk individuals. J Hepatol 2015;63:705-12.

74 Blais $P$, Lin M, Kramer JR, et al. Statins are underutilized in patients with nonalcoholic fatty liver disease and dyslipidemia. Dig Dis Sci 2016;61:1714-20.

75 Kargiotis K, Athyros VG, Giouleme 0, et al. Resolution of non-alcoholic steatohepatitis by rosuvastatin monotherapy in patients with metabolic syndrome. World J Gastroenterol 2015;21:7860-8.

76 Loomba R, Sirlin CB, Ang B, et al. Ezetimibe for the treatment of nonalcoholic steatohepatitis: assessment by novel magnetic resonance imaging and magnetic resonance elastography in a randomized trial (MOZART trial). Hepatology 2015;61:1239-50.

77 Nies VJ, Sancar G, Liu W, et al. Fibroblast growth factor signaling in metabolic regulation. Front Endocrinol (Lausanne) 2015:6:193.

$78 \mathrm{Mu}$ J, Pinkstaff J, Li Z, et al. FGF21 analogs of sustained action enabled by orthogonal biosynthesis demonstrate enhanced antidiabetic pharmacology in rodents. Diabetes 2012;61:505-12.

79 Wu X, Ge H, Lemon B, et al. FGF19-induced hepatocyte proliferation is mediated through FGFR4 activation. J Biol Chem 2010;285:5165-70.

80 Luo J, Ko B, To C, et al. Treatment with Ngm282 Significantly improves liver histopathology in a mouse model of non-alcoholic steatohepatitis (Nash). Journal of Hepatology 2015;62:S694-S.

81 Cable EE, Finn PD, Stebbins JW, et al. Reduction of hepatic steatosis in rats and mice after treatment with a liver-targeted thyroid hormone receptor agonist. Hepatology 2009:49:407-17.

82 Stanley TL, Feldpausch MN, Oh J, et al. Effect of tesamorelin on visceral fat and liver fat in HIV-infected patients with abdominal fat accumulation: a randomized clinical trial. JAMA 2014:312:380-9.

83 Nishizawa $\mathrm{H}$, Iguchi $\mathrm{G}$, Murawaki A, et al. Nonalcoholic fatty liver disease in adult hypopituitary patients with $\mathrm{GH}$ deficiency and the impact of $\mathrm{GH}$ replacement therapy. Eur J Endocrinol 2012;167:67-74.

84 Trajkovski M, Hausser J, Soutschek J, et al. MicroRNAs 103 and 107 regulate insulin sensitivity. Nature 2011;474:649-53.

85 Xu Q, Li Y, Shang YF, et al. miRNA-103: molecular link between insulin resistance and nonalcoholic fatty liver disease. World J Gastroenterol 2015;21:511-16.

86 Kowdley KV, Wilson LA, Van Natta ML, et al. Efficacy and Safety of Vitamin E in Nonalcoholic Steatohepatitis Patients With and Without diabetes: Pooled analysis from the PIVENS and FLINT NIDDK NASH CRN Trials. Hepatology 2015;62:264A-A

87 Kaempf-Rotzoll DE, Traber MG, Arai H. Vitamin E and transfer proteins. Curr Opin Lipidol 2003;14:249-54.

88 Miller ER III, Pastor-Barriuso R, Dalal D, et al. Meta-analysis: high-dosage vitamin E supplementation may increase all-cause mortality. Ann Intern Med 2005;142:37-46.

89 Abner EL, Schmitt FA, Mendiondo MS, et al. Vitamin E and all-cause mortality: a meta-analysis. Curr Aging Sci 2011:4:158-70.

90 Schürks M, Glynn RJ, Rist PM, et al. Effects of vitamin E on stroke subtypes: meta-analysis of randomised controlled trials. BMJ 2010;341:c5702.

91 Klein EA, Thompson IM Jr, Tangen CM, et al. Vitamin E and the risk of prostate cancer: the Selenium and Vitamin E Cancer Prevention Trial (SELECT). JAMA 2011:306:1549-56

92 Schwimmer JB, Lavine JE, Neuschwander-Tetri BA, et al. Cysteamine Bitartrate Delayed-Release (DR) for the Treatment of Nonalcoholic Fatty Liver Disease (NAFLD) in Children (CyNCh) Trial. Hepatology 2015;62:1398A-9A.

93 Syn WK, Choi SS, Diehl AM. Apoptosis and cytokines in non-alcoholic steatohepatitis. Clin Liver Dis 2009;13:565-80.

94 Feldstein AE, Gores GJ. Apoptosis in alcoholic and nonalcoholic steatohepatitis. Front Biosci 2005;10:3093-9.

95 Linton SD, Aja T, Armstrong RA, et al. First-in-class pan caspase inhibitor developed for the treatment of liver disease. J Med Chem 2005;48:6779-82.

96 Pockros PJ, Schiff ER, Shiffman ML, et al. Oral IDN-6556, an antiapoptotic caspase inhibitor, may lower aminotransferase activity in patients with chronic hepatitis C. Hepatology 2007;46:324-9.

97 Shiffman ML, Pockros P, McHutchison JG, et al. Clinical trial: the efficacy and safety of oral PF-03491390, a pancaspase inhibitor-a randomized 
placebo-controlled study in patients with chronic hepatitis C. Aliment Pharmacol Ther 2010:31:969-78.

98 Barreyro FJ, Holod S, Finocchietto PV, et al. The pan-caspase inhibitor Emricasan (IDN-6556) decreases liver injury and fibrosis in a murine model of non-alcoholic steatohepatitis. Liver Int 2015;35:953-66.

99 Shiffman M, Freilich B, Vuppalanchi R, et al. A Placebo-controlled, multicenter, double-blind, randomised trial of emricasan in subjects with non-alcoholic fatty liver disease (Nafld) and raised transaminases. J Hepatology 2015;62:S282-S.

100 Akriviadis E, Botla R, Briggs W, et al. Pentoxifylline improves short-term survival in severe acute alcoholic hepatitis: a double-blind, placebo-controlled trial. Gastroenterology 2000;119:1637-48.

101 Thursz MR, Richardson P, Allison M, et al. Prednisolone or pentoxifylline for alcoholic hepatitis. N Engl J Med 2015;372:1619-28.

102 Mathurin P, Louvet A, Duhamel A, et al. Prednisolone with vs without pentoxifylline and survival of patients with severe alcoholic hepatitis: a randomized clinical trial. JAMA 2013;310:1033-41.

103 Zein CO, Yerian LM, Gogate P, et al. Pentoxifylline improves nonalcoholic steatohepatitis: a randomized placebo-controlled trial. Hepatology 2011:54:1610-19.

104 Zein CO, Lopez R, Fu X, et al. Pentoxifylline decreases oxidized lipid products in nonalcoholic steatohepatitis: new evidence on the potential therapeutic mechanism. Hepatology 2012;56:1291-9.

105 Zeng T, Zhang CL, Zhao XL, et al. Pentoxifylline for the treatment of nonalcoholic fatty liver disease: a meta-analysis of randomized double-blind, placebo-controlled studies. Eur J Gastroenterol Hepatol 2014;26:646-53.

106 Du J, Ma YY, Yu CH, et al. Effects of pentoxifylline on nonalcoholic fatty liver disease: a meta-analysis. World J Gastroenterol 2014;20:569-77.

107 Xiang M, Wang PX, Wang AB, et al. Targeting hepatic TRAF1-ASK1 signaling to improve inflammation, insulin resistance, and hepatic steatosis. $J$ Hepatol 2016;64:1365-77.

108 Budas G, Karnik S, Jonnson T, et al. Reduction of liver steatosis and fibrosis with an Ask1 inhibitor in a murine model of nash is accompanied by improvements in cholesterol, bile acid and lipid metabolism. J Hepatol 2016;64:\$170.

109 Chiang SH, Bazuine M, Lumeng CN, et al. The protein kinase IKKepsilon regulates energy balance in obese mice. Cell 2009;138:961-75.

110 Reilly SM, Chiang SH, Decker SJ, et al. An inhibitor of the protein kinases TBK1 and IKK-varepsilon improves obesity-related metabolic dysfunctions in mice. Nat Med 2013;19:313-21.

111 Bertola A, Bonnafous S, Anty R, et al. Hepatic expression patterns of inflammatory and immune response genes associated with obesity and NASH in morbidly obese patients. PloS ONE 2010;5:e13577.

112 Saiman Y, Friedman SL. The role of chemokines in acute liver injury. Front Physiol 2012:3:213.

113 Marra F, Tacke F. Roles for chemokines in liver disease. Gastroenterology 2014; 147:577-94.e1.

114 Thompson M, Chang W, Jenkins H, et al. Improvements in APRI and FIB-4 fibrosis scores correlate with decreases in SCD14 in HIV-1 infected adults receiving cenicriviroc over 48 weeks. Hepatology 2014;60:424A-A
115 Friedman S, Sanyal A, Goodman Z, et al. Efficacy and safety study of cenicriviroc for the treatment of non-alcoholic steatohepatitis in adult subjects with liver fibrosis: CENTAUR Phase $2 \mathrm{~b}$ study design. Contemp Clin Trials 2016:47:356-65.

116 Lalor PF, Edwards S, McNab G, et al. Vascular adhesion protein-1 mediates adhesion and transmigration of lymphocytes on human hepatic endothelial cells. J Immunol 2002;169:983-92.

117 Weston CJ, Shepherd EL, Claridge LC, et al. Vascular adhesion protein-1 promotes liver inflammation and drives hepatic fibrosis. J Clin Invest 2015;125:501-20.

118 Jarolimek W, Charlton B. Phase 1 results from Pxs-4728a, a selective Ssao/Vap- 1 inhibitor, for the treatment of non-alcoholic steatohepatitis. J Hepatol 2015;62: S274-S5.

119 Nguyen V, George J. Nonalcoholic fatty liver disease management: dietary and lifestyle modifications. Semin Liver Dis 2015;35:318-37.

120 Promrat K, Kleiner DE, Niemeier HM, et al. Randomized controlled trial testing the effects of weight loss on nonalcoholic steatohepatitis. Hepatology 2010;51:121-9.

121 Chalasani N, Younossi Z, Lavine JE, et al. The diagnosis and management of non-alcoholic fatty liver disease: practice Guideline by the American Association for the Study of Liver Diseases, American College of Gastroenterology, and the American Gastroenterological Association. Hepatology 2012;55:2005-23.

122 Zelber-Sagi S, Kessler A, Brazowsky E, et al. A double-blind randomized placebo-controlled trial of orlistat for the treatment of nonalcoholic fatty liver disease. Clin Gastroenterol Hepatol 2006;4:639-44.

123 Harrison SA, Fecht W, Brunt EM, et al. Orlistat for overweight subjects with nonalcoholic steatohepatitis: A randomized, prospective trial. Hepatology 2009:49:80-6

124 Henao-Mejia J, Elinav E, Jin C, et al. Inflammasome-mediated dysbiosis regulates progression of NAFLD and obesity. Nature 2012:482:179-85.

125 Adar T, Ben Ya'acov A, Lalazar G, et al. Oral administration of immunoglobulin G-enhanced colostrum alleviates insulin resistance and liver injury and is associated with alterations in natural killer T cells. Clin Exp Immunol 2012;167:252-60.

126 Mizrahi M, Shabat Y, Ben Ya'acov A, et al. Alleviation of insulin resistance and liver damage by oral administration of Imm124-E is mediated by increased Tregs and associated with increased serum GLP-1 and adiponectin: results of a phase I/I clinical trial in NASH. J Inflamm Res 2012;5:141-50.

127 Vrieze A, Van Nood E, Holleman F, et al. Transfer of intestinal microbiota from lean donors increases insulin sensitivity in individuals with metabolic syndrome. Gastroenterology 2012;143:913-6.e7.

128 Fernandes $\mathrm{P}$, Hashiguchi T, Fujii M, et al. Anti-NASH Effects of Solithromycin in NASH-HCC Mouse Model. Gastroenterology 2014;146:S145-6.

129 Fernandes P. Mechanism of Action of the Anti-NASH effects of Solithromycin in a Predictive NASH HCC Mouse Model. Hepatology 2015;62:1301A-A.

130 Barry-Hamilton V, Spangler R, Marshall D, et al. Allosteric inhibition of lysyl oxidase-like-2 impedes the development of a pathologic microenvironment. Nat Med 2010;16:1009-17.

131 Traber PG, Zomer E. Therapy of experimental NASH and fibrosis with galectin inhibitors. PloS ONE 2013:8:e83481. 